\title{
Global Perspectives on Palliative Care for Cancer Patients: Not All Countries Are the Same
}

\author{
Jeannine M. Brant ${ }^{1}$ (D) Michael Silbermann ${ }^{2}$ \\ Accepted: 12 February 2021 / Published online: 8 April 2021 \\ (C) The Author(s), under exclusive licence to Springer Science+Business Media, LLC, part of Springer Nature 2021
}

\begin{abstract}
Purpose of the Review

The integration of cancer-related palliative care is essential to holistic, quality cancer care. While some similarities exist between countries, this manuscript will focus on five differences that impact palliative care for cancer patients including the epidemiology of cancer and related symptoms, cancer-specific integration into care, palliative care education, economic development of the country, and cultural and religious differences.
\end{abstract}

\section{Recent Findings}

The epidemiology of cancer varies around the world resulting in variable symptoms and the need for individualized approaches to palliative care. While palliative care is integrated in some countries, it is lacking in over half of the world, and specific integration into cancer care is virtually absent. Education and training are the key to expansion, and yet oncology-focused palliative care education is lacking or is not well-reported in the literature. To complicate this global lens even further are the economic disparities that exist. Low-to-middle-income countries (LMICs) are resource poor and have the fewest resources and least amount of integration, and yet patients with advanced cancer are over-represented in these countries. Essential to cancerrelated palliative care is a tailored approach that addresses cultural and religious differences around the globe.

\section{Summary}

Palliative care is developing around the globe and yet palliative care specific for cancer patients is in its infancy. Cancer care professionals should (1) understand the epidemiologic differences that exist globally and the impact this has on palliative care, (2) integrate palliative care into the cancer care arena, (3) provide cancer-specific palliative education focused on the cancer trajectory from diagnosis through survivorship and end of life, (4) advocate for LMICs, which suffer from a lack of resources and services, and (5) understand cultural and religious differences that exist to provide holistic and sensitive cancer-related palliative care.

Keywords Palliative care $\cdot$ Cancer $\cdot$ Disparities $\cdot$ Low-to-middle-income countries $\cdot$ Global health

\section{Introduction}

Cancer is the second leading cause of death around the world. A recent systematic review estimated that approximately 17

This article is part of the Topical Collection on Palliative Medicine

Jeannine M. Brant

jbrant@billingsclinic.org

1 Billings Clinic, 2651 North Bridger Drive, Billings, MT 59102, USA

2 Middle Eastern Cancer Consortium, Haifa, Israel million people are diagnosed with cancer each year and 9.6 million will die from the disease [1]. With this comes a desperate need for adequate palliative care, to support patients throughout cancer treatment and through survivorship and end of life care. Palliative care is an essential component of cancer care around the globe and employs an interdisciplinary approach to relieve suffering through the management of pain and symptoms caused by cancer and its treatment [2].

For over a decade, experts have suggested that a global approach is essential to the development of palliative care worldwide in terms of leadership, sharing of knowledge, and advocacy. Ongoing efforts continue to better integrate 
palliative care into cancer care, and it is apparent that one size does not fit all. While some similarities exist in palliative cancer care around the world, vast differences exist in five primary areas: (1) the epidemiology of cancer and related symptoms experienced, (2) cancer-specific integration into care, (3) palliative care education, (4) economic development of the country, and (5) cultural and religious differences that call for a tailored approach to care. This manuscript will discuss recent evidence on palliative cancer care around and five differences that impact care. Future directions in research and practice will be recommended.

\section{Global Similarities}

While it is sometimes easiest to focus on differences between countries, three commonalities exist within global palliative cancer care that are foundational to this discussion. First, the primary goal of palliative care is universal: to relieve pain and suffering and to improve quality of life, [2] which many refer to as a human right $[3 \cdot \bullet, 4]$. While this is globally similar, the degree and means to accomplish this may be very different. Secondly, the interdisciplinary team is foundational to the delivery of palliative care. Employing a team is what separates integrated palliative care to a lone clinician providing supportive cancer care. And third, the core of all societies around the world is the family unit, which holds special meaning for the delivery of cancer palliative care around the globe. [5-8] This common ground infers that we as a human race are often more similar than we are different. And yet, our circumstantial and individual differences create a rich diversity in who we are as people, which calls for a tailored approach to palliative care.

\section{Epidemiology of Cancer Around the World}

The epidemiology of cancer varies around the world, and is directly impacted by a country's aging population, lifestyle choices, and infectious-related cancers [9]. GLOBOCAN estimates the incidence and mortality of 36 cancers in 185 countries, and most recent evidence indicates a growing number of cancers related to behaviors such as smoking and diet and a decrease in infection-related cancers [10]. Figure 1 depicts a map of the global incidences of cancer in males and females. These rates directly impact palliative care in several ways. First, some cancers such as lung cancer are often diagnosed at later stages, especially in developing countries, and symptom burden and the need for palliative care are often high. Second, symptom clusters exist among certain types of cancer. For example, dyspnea and cough are commonly experienced in patients with lung cancer, the predominant cancer in Asian men [11]. Patients with gastrointestinal cancer can experience 13 co-occurring symptoms including nausea, vomiting, and pain, [12] which impacts patients with liver cancer, the most common cancer in 13 countries. For women, breast cancer is the most common cancer around the world. Also important to mention is cervical cancer, which is the most common cancer in 28 countries [10].

Because the epidemiology of cancer is highly variable around the world, it is likely that symptoms may also vary, and yet a lack of global studies exist on the symptom experience. A systematic review of international research in palliative care found in the 184 studies examined, the majority were observational studies or program evaluations [13•]. Research that representatively focuses on patient symptoms, outcomes, and interventions is relatively absent. A handful of studies do exist.

One recent meta-analysis of 36 studies in LMICs was recently published that reported on lymphedema of the arm secondary to breast cancer treatment $(n=31)$ and leg following gynecological cancer treatment $(n=5)$. High variability existed in circumference measurements, and standardizing measurement was recommended to better understand and report the problem globally [14]. Odonkor and colleagues [15] published a systematic review comparing the quality of cancer pain management trials in Africa, Europe, and North America. Only three of 18 trials were from Africa (Egypt), and authors acknowledged the disproportionate representation of trials globally [15].

\section{Palliative Care Integration into Cancer Care}

Palliative care services exist in approximately 136 of the world's 234 countries. The degree of integration is highly variable, however, and resources are country dependent. Lynch et al. [16] mapped palliative care development around the world, and found levels ranging from no activity to advanced integration, which emphasized some of the global disparities that exist globally, further emphasizing that not all countries are alike. Europe, Australia, Canada, and the USA have the most advanced integration, whereas several areas in Africa and parts of Asia and South America are lacking services. It should be noted that specific integration of cancerrelated palliative care was not addressed in Lynch's work.

A handful of papers have been written that describe integration of palliative services into cancer care. One systematic study examined 60 guidelines and 14 clinical pathways in Europe and found that $80 \%$ used a holistic approach and $66 \%$ employed palliative care interventions to alleviate suffering [17]. In a survey of 15 Middle Eastern countries $(n=773$ respondents), $54.7 \%$ reported having a palliative care consultation service in their organization and $41.4 \%$ had a palliative care unit [18]. While cancer-related palliative care was not addressed specifically, many of the authors and respondents of this paper work are directly in cancer care. Another European systematic review examined 14 studies of palliative care integration, but only four of these studies included 

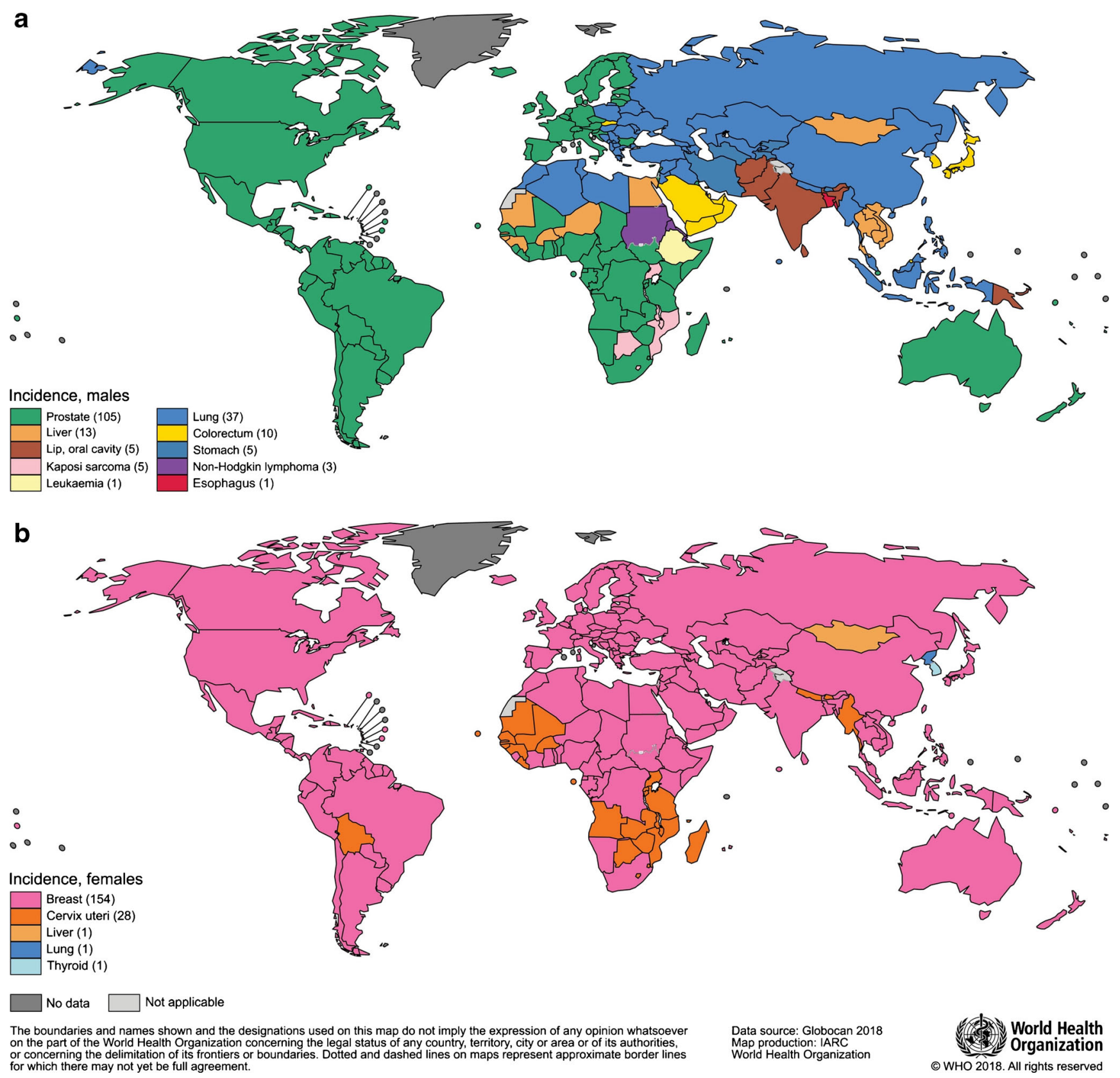

Fig. 1 Global diversity of cancer incidences in males and females

patients with cancer and two noted a combined chronic disease and cancer model [19]. Outcomes were positive in that patients who received palliative care had fewer hospitalizations and better quality of life. Japan has also reported successful integration of palliative care into cancer care [20].

It is obvious that palliative care integration is lacking in many countries around the world. In a survey of 29 countries on six continents ( $n=749$ respondents), home health nursing was the only supportive care available, especially in remote areas [21]. Overall, the integration of palliative care worldwide is still growing. And specific integration for cancer patients appears to be in early stages in more developed countries, but a lack of evidence exists for other parts of the world. This is complicated in that integrating palliative care into cancer care is also not well-defined, but rather a heterogeneously defined concept [22]. Further defining integration will aid countries globally in determining successful integration of palliative care into cancer care.

\section{Cancer-Related Palliative Care Education}

The ability to successfully integrate palliative care for cancer patients into a country is highly dependent on access to both 
professional and community education. While a plethora of global palliative care educational programs exist, the majority are general and not specific to cancer care. The Journal of Pain and Symptom Management devoted a 2018 supplement to the International Palliative Care Initiative, which provided $\$ 25$ million to the global advancement of palliative care including professional education and curriculum and leadership development [3]. The End of Life Nursing Education Consortium is available globally at http://www.aacn.nche. edu/elnec/about/fact-sheet, and an oncology-specific course exists through this curriculum; however, reports of global use and course outcomes are lacking in the literature. The Education Physician in End of Life Care-Oncology (EPECO), sponsored by the National Cancer Institute, has been integrated into undergraduate and postgraduate educational curricula in Ukraine [23••], but other reports of integration are lacking. One systematic review of 14 palliative oncologic care curricula for providers in resource-limited and underserved communities referenced programs in Argentina, Uganda, Kenya, Australia, Germany, the USA, and some combined countries. Education topics included palliative care principles, pain and symptom management content, and communication skills, primarily presented in a didactic format with some experiential learning [24]. Only one program specifically mentioned oncology-specific content.

It is possible that more cancer-specific content has been integrated into palliative care education globally, and yet this information is not disseminated. Or it is possible that dissemination has occurred in other venues, such as books and book chapters, that are not captured well in literature reviews. Perhaps some of the best examples of this work come from professor Silbermann and his work with countries around the world. Books include Palliative Care: Perspectives, Practices, and Impact on Quality of Life: A Global View [25] and Cancer Care in Countries and Societies in Transition [26], which include chapter exemplars on the integration of palliative care into oncology.

\section{Economic Development}

Cancer is a disease that affects all areas of the world, and yet $70 \%$ of deaths from cancer occur in low-to-middle-income countries (LMICs). This is primarily due to late diagnosis, where curative treatment is not an option, and hence, palliative care is desperately needed [2]. According to a Global Palliative Care Resource-Stratified Guideline by the American Society Clinical Oncology, all patients with cancer who cannot receive curative treatment should receive palliative care [27•]. While palliative care services exist in over half of the world's countries, these programs are primarily located in high-income countries and are lacking in areas where patients may be in most need $[16,28]$.
Palliative care efforts and research are underway in LMICs and resource-limited settings around the globe, and yet a sparsity of data exist. One systematic review of palliative care intervention outcomes in low-resource countries identified only 18 studies, and only five of which included patients with cancer [29]. The majority of these focused on the management of pain, and outcomes were positive. A recent systematic review of 18 studies appraised improvement strategies of LMICs to increase access to cancer treatments and palliative care. The majority were case reports; research was lacking. Studies did identify facilitators to be stakeholder engagement, financial support, supportive learning environment, and community networks, whereas barriers were lack of human resources, financial constraints, and limited infrastructure [30 $0^{\bullet}$.

Possibly the most disturbing aspect of this economic gap is the fact that palliative care is cost-effective. A review of 10 papers that evaluated cost-effectiveness of palliative care in LMICs found that the four studies that conducted cost-effective analyses found both financial savings and positive patient outcomes [31]. Overall, the authors reported that low-cost delivery of palliative care in LMICs is possible, and that palliative care is a less costly option compared to other types of treatment.

\section{Culture and Religion}

The most notable global differences in palliative care for cancer patients are cultural and religious diversity between countries and geographic regions. Both are worthy of discussion and imply a tailored plan for palliative care. One of the most common beliefs that impact both cancer and palliative care is fatalism, the perception that cancer, pain, and suffering are inevitable $[32,33]$. These beliefs may be spiritually or culturally based or could be related to a lack of knowledge about treatment and palliative care options [34]. It may be more prominent for some regions in the world such as Asia [35, 36] or in some cultural and religious groups [37-39]. A "natural fate" is a predominant belief in Buddhism and encourages patients to confront suffering while waiting until death comes [40]. China is predominantly a Buddhist society, and higher fatalism scores exist.

Not only fatalism, but fear of cancer, which includes an avoidance of discussing the illness, is common among some cultures. Fear can be related to fatalism and in the belief that cancer is not curable. Superstition can be another part of the fear and some cultural groups continue to think that cancer is contagious, especially in parts of Africa [41] and even in US American Indians. When working with an American Indian medicine man, he stated, talking about cancer will bring bad medicine to my people [42]. For Middle Eastern and other cultures, it is also common not to disclose a cancer diagnosis. Doing so is thought to have a 
Table 1 Influence of religion on palliative care

\begin{tabular}{|c|c|c|}
\hline $\begin{array}{l}\text { Religion or } \\
\text { spiritual } \\
\text { foundation }\end{array}$ & Beliefs about pain & Implications \\
\hline Buddhism [40] & $\begin{array}{l}\text { - Suffering is a part of human existence. } \\
\text { - Pain should be accepted; suffering with pain leads to spiritual } \\
\text { growth. } \\
\text { - Pain experience influenced by Buddhist beliefs on the Four } \\
\text { Noble Truths which has to do with why people suffer. } \\
\text { - Buddhism teaches that the pain is a "natural fate" that patients } \\
\text { should confront while waiting until death comes. These } \\
\text { perspective patients with higher fatalism scores consider pain as } \\
\text { an inevitable experience, be hesitant to use analgesics, and a } \\
\text { willingness to endure pain for months. }\end{array}$ & $\begin{array}{l}\text { - Healthcare professionals can assist patients to focus mentally } \\
\text { and not on physical suffering so that patients are not too } \\
\text { focused on their disease. }\end{array}$ \\
\hline Christianity [51] & $\begin{array}{l}\text { - Pain medications accepted if it provides comfort to the patient } \\
\text { - Less concern mentioned in the literature for opioids and a } \\
\text { decrease in consciousness } \\
\text { - Catholic principle of "double effect" accepted for physical pain; } \\
\text { e.g., benefits of opioids should outweigh the side effects of } \\
\text { sedation and potential loss of consciousness }\end{array}$ & $\begin{array}{l}\text { - Focus on the benefit of patient comfort while discussing } \\
\text { potential for untoward side effects from opioids }\end{array}$ \\
\hline Hinduism [50] & $\begin{array}{l}\text { - Belief in Karma is central to pain interpretation and acceptance } \\
\text { of analgesics. } \\
\text { - Pain considered the result of Karma; the natural consequence of } \\
\text { inappropriate actions from this life or from a past life } \\
\text { - Pain not considered good or bad, but rather, that which is given } \\
\text { from God }\end{array}$ & $\begin{array}{l}\text { While all attempts should be made to alleviate suffering, } \\
\text { Hinduism accepts the outcome that pain may not be relieved. }\end{array}$ \\
\hline Islam $[34,49,51]$ & $\begin{array}{l}\text { - Pain and suffering are perceived as a motive to seek God's } \\
\text { forgiveness or expiation for wrongs that were committed. } \\
\text { - Islam encourages Muslims to accept that whatever things } \\
\text { happen, whether good or bad (e.g., pain) is from God. }\end{array}$ & $\begin{array}{l}\text { - May choose to avoid use of pharmacologic agents until the } \\
\text { intensity of their pain is increased and becomes unbearable due } \\
\text { to thoughts that pain is God's will and hence it must be } \\
\text { accepted. } \\
\text { - While all things are from God, is permissible to seek relief of } \\
\text { pain and accept treatment. } \\
\text { - Pain management can include reading the holy Quran, making } \\
\text { dua (an act of supplication), reciting prayers, and seeking } \\
\text { support of the Islamic community. }\end{array}$ \\
\hline Judaism $[51,52]$ & $\begin{array}{l}\text { - Pain medications accepted for comfort } \\
\text { - Concern for decreased level of consciousness not as commonly } \\
\text { mentioned as with other religions } \\
\text { - Principle of "double effect" may be accepted from a Jewish } \\
\text { perspective. }\end{array}$ & $\begin{array}{l}\text { - Provide analgesics for comfort; discuss potential side effects of } \\
\text { sedation with the patient and family to determine acceptance }\end{array}$ \\
\hline
\end{tabular}

Reprinted from Seminars in Oncology Nursing, 35(3), Can, G., Mushani, T., Rajhi, B. H. A., \& Brant, J. M., The Global Burden of Cancer Pain, p. 317, 2019, with permission from Elsevier

negative impact on the patient [43]. And when cancer or end of life is disclosed, it is not uncommon for families to socially isolate or abandon their loved one due to social stigma [6]. Overall, cancer can be considered fearful and a cultural taboo in many cultures, making discussion of cancer and palliative care a challenge [44].

The use of traditional medicine is another common and yet culturally diverse component of palliative care for cancer patients. One systematic review of 39 papers that examined palliative care practices in indigenous persons found that patients desired integration of traditional medicine and spiritual healers into their care [45]. Palliative care clinicians need to better understand how to incorporate these practices safely into cancer care.

Decision-making, which is an important component of palliative care, may be very different depending on cultural. In some cultures, the patient is left out of the conversation, and palliative care decisions are instead discussed with the whole family or with the patient's children [36, 46]. This is common in both Chinese and Arab societies. Involving the family is important in the overall plan of care [47]. This varies from most Western cultures, in which individual independence is important, and decisions are left to the patient, although the degree of family engagement may greatly vary [46]. One area of decision-making involves ventilator withdrawal. While ventilator use is not common for cancer patients receiving palliative care in Western culture, it may be common in other parts of the world, especially in children. One systematic review of 25 papers from nine countries found that practices vary greatly. The US and Western Europe more commonly withdraw ventilation, whereas in Japan, it is seen as life- 
shortening and against the law. In Slovenia, ventilators are never or rarely withdrawn [48].

\section{Religion}

Significant religious differences exist around the world with various perspectives on life, death, and suffering, which includes cancer. Table 1 includes information of the world's most common religions/spiritual foundations, beliefs, and implications for palliative care [34, 40, 49-52]. The use of opioids and other sedatives used in palliative care deserves thoughtful attention through a religious lens as impacting a person's level of alertness or disrupting mental orientation is against some religious beliefs [51]. However, individual variation occurs within each religion, and so it is important for the palliative care team to individually assess the patient and not rely solely on religious directives.

\section{Discussion and Future Directions}

Palliative care for cancer patients is evolving globally. While not all countries are alike, there is hope on the horizon for better treatments, earlier detection of cancer, improved survival, increased access to rigorous palliative care education, economic development within countries to allow for optimal palliative care, and individualized cultural and religious integration into care.

Regarding cancer prevalence, between the years 2010 and 2020, the number of new cancer cases in the USA has risen by approximately $24 \%$ in men, to more than one million cases per year, and approximately $21 \%$ in women, to more than 900,000 cases per year. Over the next decade, the cancer incident rate is expected to stay about the same, but the number of new cases is expected to go up, mostly due to the aging Caucasian population and the growing population of people of color. In highincome countries, fewer Caucasian women are expected to die from breast, cervical, and colorectal cancers due to better treatments and the fact that they are more likely to get screened for these cancers. More access to high-quality healthcare has also led to an increase in the rate of survival and fewer deaths from colorectal cancer and, to a lesser extent, from prostate cancer and female breast cancer [53]. This growing incidence in the USA will likely ripple across the globe, along with improvements in early detection and better treatment.

Integrating palliative care, not only into cancer care but also into the landscape of healthcare in each country around the world, will be more challenging. Last year, in the 2019 World Cancer Leaders' Summit in Kazakhstan, HRH Princess Dina Mired, President of the Union International Cancer Control (UICC), declared, "Governments around the world have recognized how important it is to deliver universal health coverage (UHC), including accelerating efforts to tackle cancer. But while the recent adoption of the Political Declaration on UHC in New York was a very important step, it is only the beginning of a much longer journey. In many countries people have lost trust in their health systems. Regaining their trust and investing better and smarter is key to making progress." [54]

Indeed, not all countries enjoy modern palliative care services and, accordingly, the estimated 5-year net survival rate, globally, differs significantly. In high-income countries, the survival rate for 11 types of cancer is 12 times higher than that in lower-income countries. Improving the quality of care, including the full continuum of palliative care services, could significantly improve 5-year net survival rates in lower- and middle-income countries [55].

Palliative care and pain relief are some of the most neglected dimensions of global health today. They are barely even afterthoughts in discussions regarding the scope of universal healthcare. Even high-income countries with advanced health services struggle to provide access to comprehensive palliative care programs. Cancer treatment may be financially covered, but healthcare coverage for palliative care may be limited. If the professional, public and political communities cannot agree on palliative care's place in society, introducing this element into discussions of what should be included within UHC is unlikely to be helpful or productive [56].

To date, palliative care at the highest level of provision is available for only $14 \%$ of the global population and is concentrated in European countries. An $87 \%$ global increase in serious health-related suffering amenable to palliative care interventions is predicted by 2060 . With an increasing need, palliative care is not reaching the levels required by at least half of the global population [57]. Pediatric palliative care at the highest level of provision is available in just 21 countries, accounting for fewer than $10 \%$ of the global population aged 20 and under. It is concentrated in high-income settings, while the majority of the global need for such care occurs in low- and middle-income countries [58]. Significant work is needed to fully integrate cancer-related palliative care into countries around the world.

LMICs deserve specific recognition in this discussion, as the provision of palliative care to cancer patients is affected by a wide range of challenges. Personal- and healthcare systemrelated issues create obstacles. Overall, insufficient knowledge, poor awareness, negative attitudes and beliefs of patients, families, general public, and healthcare providers present crucial personal challenges to the provision of palliative care [59••].

Palliative care education is recommended as the first step in overcoming these challenges. In oncology, physicians often do not consider the importance of person-centered care in their daily clinical practice, and how it relates to their training. Most oncologists struggle with choosing the right words. Such difficulties are a consequence of their limited education in palliative care during their oncology fellowships. Moreover, few have training or mentorship in communication skills, as oncologists are trained to be medicine-oriented, not person-oriented. Even in the leading 
high-income countries, communication issues between patients and their caregivers concerning preferences and prognoses serve as a barrier to satisfactory care. Proper communication requires skills that, unfortunately, are not yet included in either the physician or nursing curriculum. The current education efforts have still not produced the needed cultural- and medical-political changes that would bring about the optimal practice of palliative care principles, globally. Furthermore, in the USA, rarely has an oncologist been recommended a career focused on symptom management or palliation. Therefore, what is missing and what is needed are training programs that allow tailored experiences that blend the focus on both treating the disease and supporting the people affected by it. The key to this is integration. With oncology training focused on treatment, clinical trials, and drug development, it is not surprising that palliative care is still not integrated into cancer care [60]. A more recent publication from the USA revealed that, generally, there are positive attitudes toward primary palliative care education; however, there are still significant numbers of MD trainees with no little to no exposure and heterogeneity in the content and delivery of primary palliative care education across specialties [61].

The interdisciplinary team should also be further developed globally. But sadly, in the most recent WHO report in 2020 on Cancer Profile, palliative care personnel were not even mentioned in its workforce list. However, in developed countries and, even more so, in developing countries, the primary palliative care team is essential. For patients and their caregivers in the community, a critical factor is the access to skilled home-care nursing 24/7. Specialist palliative care teams, who mostly work in hospitals, are a valuable source and collaborative partners for primary care teams. Thus, involving the specialist palliative care team, as needed, enables the primary care team to remain the main point of contact for the patient [62]. And yet in most developing countries, cancer patients in need of palliative care are at home, due to transportation difficulties or limited income, and cannot access proper care or purchase-required medications. Integrating palliative care into primary care services is a recommended strategy for improving the accessibility of palliative care to those living in remote areas. There remains a lack of available cost data, which creates a hindrance to introducing and estimating palliative care costs [59••].

The ability for palliative care teams to reach all populations, however, is another major challenge. One of the main reasons for the discrepancy in the quality of palliative care services in developed countries versus developing countries is the fact that in most Western countries, including Australia, New Zealand, and Japan, palliative care has been institutionalized and is carried out in hospices and home-hospices, whereas in almost all developing countries, cancer patients end their lives at home without the optimal care that modern palliative care services are able to provide to suffering patients and their families [63].

By and large, challenges that hinder the provision of palliative care differ significantly between developed and developing countries - in terms of scope, context, culture, and religious beliefs. However, there are also some similarities, such as the most common obstacles, including the lack of a properly trained workforce. Furthermore, negative attitudes regarding palliative care and death, opioid phobia, and families' feelings of alienation and isolation are the most common challenges in developing countries, while misperceptions of the general public and healthcare personnel equating "palliative care" with "end-of-life care" are the main challenges in developed countries.

It is evident that spirituality, as part of being human, emerges in the intersubjective encounter between the caregiver/nurse and the human being/patient as a place of mobilization and inner strength, capable of generating resilience through faith in times of illness. Spiritual care is recognized by the nursing profession as opportune, especially in the context of hospitalization, where human vulnerability strikes people in a marked way, favoring the bond and exchange of positive experiences in the healthcare process [64]. However, the theme of "spirituality" and its practices in the daily work of healthcare teams are still a challenge which needs to be better-addressed and worked on during the process of continuing education for these professionals in order for them to develop the skills and confidence to welcome this demand. These may include mindful listening, music, prayer, and accessibility to religious leaders and practices for directing the mobilization of faith and hope to the patient/family; transpersonal caring (the ability to center consciousness and intentionality on caring, healing, and wholeness, rather than on disease, illness and pathology) is also capable of subsidizing spiritual care by offering a care model to the whole mind-body-soul [64]. It is clear that in both developed and developing countries, access to religious and spiritual resources is important during cancer treatment and incorporating them into cancer care may be especially important to patients that identify as religious. Religion and spirituality need should be addressed as part of the cancer care plan [65].

Compassion fatigue is a growing concern of palliative care professionals around the world. Caring for the patient is not enough; the palliative care team also deserves spiritual care. A recent study from the US National Institutes of Health, Mayo Clinic, Northwestern University, and the Karolinska Institute of Sweden revealed the effectiveness of a brief mindfulnessbased program during work hours for reducing stress and anxiety with a mixed group of healthcare professionals [66]. In recent years, the Middle East Cancer Consortium's Research Group in Integration Oncology (MERGIO) has been active in promoting and training healthcare providers from the Middle East in palliative care principles, concepts, and techniques, including mindful touch [67]. Palliative care education and training should incorporate these types of approaches that care for the team and promote wellness.

Last but not least, in addition to its remarkable advantages in patient care, palliative medicine needs more clinical and basic research in order to upgrade its professional status, both 
in developed and developing countries $[68,69]$. Research that focuses on patient-reported outcomes, supportive care interventions, and the impact of the palliative care team on patient outcomes is essential. Research has potential to drive policy discussions and increase awareness of the gaps that exist in palliative care for cancer patients around the globe. While not all countries are alike, it is important that the world strives for palliative care for cancer patients that relieves suffering, integrates an interdisciplinary approach with a family focus, and remains tailored for individual differences that exist.

\section{Conclusion}

Palliative care is developing around the globe and yet palliative care specific for cancer patients is in its infancy. Cancer care professionals should recognize the gaps that exist and develop strategies to successfully integrate palliative care into cancer care. To begin this integration, clinicians should understand the epidemiologic cancer differences that exist globally and the impact this has on palliative care. Next, clinicians need to examine their personal healthcare clinics and environments to begin asking how they can facilitate this integration. This could involve obtaining more education on palliative care or could involve proposing models and for integration. And finally, as global citizens of the world, we need to recognize the needs of LMICs, who suffer from a lack of resources and services, and develop partnerships that will help to grow palliative care in these disparate countries.

\section{Declarations}

Conflict of Interest Jeannine M. Brant is a speaker's bureau for Daiichi Sankyo for Enhertu, a breast cancer pharmacologic agent. Michael Silbermann declares no conflict of interest.

Human and Animal Rights and Informed Consent This article does not contain any studies with human or animal subjects performed by the authors.

\section{References}

Papers of particular interest, published recently, have been highlighted as:

- Of importance

•- Of major importance

1. Global burden of disease cancer collaboration. Global, regional, and national cancer incidence, mortality, years of life lost, years lived with disability, and disability-adjusted life-years for 29 cancer groups, 1990 to 2017: a systematic analysis for the global burden of disease study. JAMA Oncol. 2019;5(12):1749-68.
2. World Health Organization. Palliative care. WHO. 2017 http:// www.who.int/cancer/palliative/definition/en/. Published 2017. Accessed 9 Jan 2017.

3.• Callaway MV, Foley KM. The international palliative care initiative. J Pain Symptom Manage. 2018;55(2S):S1-5 This paper sets the stage for the International Palliative Care Initiative (PICI) and highlights the partners and strategies of this important initiative. The authors provide the world with a vision for palliative care and a direction for future efforts and describes the supplemental papers that follow in the special issue of the Journal of Pain and Symptom Management.

4. Kaasa S, Loge JH, Aapro M, Albreht T, Anderson R, Bruera E, et al. Integration of oncology and palliative care: a Lancet Oncology Commission. Lancet Oncol. 2018;19(11):e588-653.

5. Wagner JM. Shifting family structure: theoretical perspectives of worldwide population and family composition. In: Stevenson EL, Herschberger PE, editors. Fertility and Assisted Reproductive Technology (ART): Theory, Research, Policy, and Practice for Health Care Practitioners. New York: Springer; 2016. p. 15-28.

6. Abdullah R, Guo P, Harding R. Preferences and experiences of Muslim patients and their families in Muslim-majority countries for end-of-life care: a systematic review and thematic analysis. J Pain Symptom Manage. 2020;60:1223-1238.e4.

7. Bates MJ, Mphwatiwa T, Ardrey J, Desmond N, Niessen LW, Squire SB. Household concepts of wellbeing and the contribution of palliative care in the context of advanced cancer: a photovoice study from Blantyre, Malawi. PLoS One. 2018;13(8):e0202490.

8. Mcllfatrick S, Muldrew DHL, Hasson F, Payne S. Examining palliative and end of life care research in Ireland within a global context: a systematic mapping review of the evidence. BMC Palliat Care. 2018;17(1):109.

9. American Cancer Society. Cancer facts and figures 2020. American Cancer Society. Cancer Facts and Figures 2020 Web site. 2020 https://www.cancer.org/research/cancer-facts-statistics/all-cancerfacts-figures/cancer-facts-figures-2016.html. Accessed 2 June 2020

10. Bray F, Ferlay J, Soerjomataram I, Siegel RL, Torre LA, Jemal A. Global cancer statistics 2018: GLOBOCAN estimates of incidence and mortality worldwide for 36 cancers in 185 countries. CA Cancer J Clin. 2018;68(6):394-424.

11. Russell J, Wong ML, Mackin L, Paul SM, Cooper BA, Hammer M, et al. Stability of symptom clusters in patients with lung cancer receiving chemotherapy. J Pain Symptom Manage. 2019;57(5): 909-22.

12. Han CJ, Reding K, Cooper BA, Paul SM, Conley YP, Hammer M, et al. Stability of symptom clusters in patients with gastrointestinal cancers receiving chemotherapy. J Pain Symptom Manage. 2019;58(6):989-1001 e1010.

13. Clark J, Gardiner C, Barnes A. International palliative care research in the context of global development: a systematic mapping review. BMJ Support Palliat Care. 2018;8(1):7-18 This systematic review identifies and thematically synthesizes all international palliative care research, which are studies that involve two or more countries. This allows the global audience to identify gaps in current research and set priorities for future studies.

14. Torgbenu E, Luckett T, Buhagiar MA, Chang S, Phillips JL. Prevalence and incidence of cancer related lymphedema in low and middle-income countries: a systematic review and meta-analysis. BMC Cancer. 2020;20(1):604.

15. Odonkor CA, Kim G, Erdek M. Global cancer pain management: a systematic review comparing trials in Africa, Europe and North America. Pain Manag. 2017;7(4):299-310.

16. Lynch T, Connor S, Clark D. Mapping levels of palliative care development: a global update. J Pain Symptom Manage. 2013;45(6):1094-106.

17. Van Beek K, Siouta N, Preston N, et al. To what degree is palliative care integrated in guidelines and pathways for adult cancer patients 
in Europe: a systematic literature review. BMC Palliat Care. 2016;15:26.

18. Silbermann M, Fink RM, Min SJ, Mancuso MP, Brant J, Hajjar R, et al. Evaluating palliative care needs in Middle Eastern countries. J Palliat Med. 2015;18(1):18-25.

19. Siouta N, Van Beek K, van der Eerden ME, et al. Integrated palliative care in Europe: a qualitative systematic literature review of empirically-tested models in cancer and chronic disease. BMC Palliat Care. 2016;15:56.

20. Uneno Y, Sato K, Morita T, Nishimura M, Ito S, Mori M, et al. Current status of integrating oncology and palliative care in Japan: a nationwide survey. BMC Palliat Care. 2020;19(1):12.

21. Brant JM, Fink RM, Thompson C, Li YH, Rassouli M, Majima T, et al. Global survey of the roles, satisfaction, and barriers of home health care nurses on the provision of palliative care. J Palliat Med. 2019;22(8):945-60.

22. Hui D, Kim YJ, Park JC, Zhang Y, Strasser F, Cherny N, et al. Integration of oncology and palliative care: a systematic review. Oncologist. 2015;20(1):77-83.

23.• Callaway MV, Connor SR, Foley KM. World Health Organization public health model: a roadmap for palliative care development. J Pain Symptom Manage. 2018;55(2S):S6-S13 This paper provides a roadmap for countries to successfully develop palliative care, with considerations for governmental and nongovernmental organizations. Considerations for palliative care policy, legislation, pain and symptom management, education, and palliative care services are included. Working groups for each key area report strategies and indicators for success.

24. Xu MJ, Su D, Deboer R, Garcia M, Tahir P, Anderson W, et al. Palliative oncologic care curricula for providers in resource-limited and underserved communities: a systematic review. J Cancer Educ. 2019;34(2):205-15.

25. Silbermann M. Palliative care: perspectives, practices, and impact on quality of life: a global view. New York: Nova Science Publishers, Inc.; 2017.

26. Silbermann ME. Cancer care in countries and societies in transition. Switzerland: Springer; 2016.

27. Osman H, Shrestha S, Temin S, Ali ZV, Cleary JF. Palliative care in the global setting: ASCO resource-stratified practice guideline summary. J Oncol Pract. 2018;14(7):431-6 This guideline summary summarizes the commitment from the American Society of Clinical Oncology (ASCO) to integrate palliative care within oncology settings around the globe. An ASCO expert panel examines the progress made in palliative care integration, but highlights the challenges which are disproportionate in low-tomiddle-income countries. ASCO provides recommendations for personnel, training, workforce, and timing of palliative care in resource-constrained countries.

28. World Health Organization. Global atlas of palliative care at the end of life. Geneva, Switzerland: WHO; 2014.

29. Potts M, Cartmell KB, Nemeth L, Bhattacharjee G, Qanungo S. A systematic review of palliative care intervention outcomes and outcome measures in low-resource countries. J Pain Symptom Manage. 2018;55(5):1382-1397.e1387.

30. Donkor A, Luckett T, Aranda S, Phillips J. Barriers and facilitators to implementation of cancer treatment and palliative care strategies in low- and middle-income countries: systematic review. Int $\mathrm{J}$ Public Health. 2018;63(9):1047-57 The authors appraise strategies adopted by low-to-middle-income countries to increase access to cancer treatments and palliative care. Facilitators identified were stakeholder engagement, financial support, a supportive learning environment, and establishment of community networks. Barriers were lack of human and financial resources and limited infrastructure.

31. Reid EA, Kovalerchik O, Jubanyik K, Brown S, Hersey D, Grant L. Is palliative care cost-effective in low-income and middle-income countries? A mixed-methods systematic review. BMJ Support Palliat Care. 2019;9(2):120-9.

32. Gunnarsdottir S, Sigurdardottir V, Kloke M, Radbruch L, Sabatowski R, Kaasa S, et al. A multicenter study of attitudinal barriers to cancer pain management. Support Care Cancer. 2017;25(11):3595-602.

33. Gunnarsdottir S, Donovan HS, Serlin RC, Voge C, Ward S. Patientrelated barriers to pain management: the Barriers Questionnaire II (BQ-II). Pain. 2002;99(3):385-96.

34. Al-Atiyyat NMH, Vallerand AH. Patient-related attitudinal barriers to cancer pain management among adult Jordanian patients. Eur J Oncol Nurs. 2018;33:56-61.

35. Chen CH, Tang ST, Chen CH. Meta-analysis of cultural differences in Western and Asian patient-perceived barriers to managing cancer pain. Palliat Med. 2012;26(3):206-21.

36. Xu X, Luckett T, Wang AY, Lovell M, Phillips JL. Cancer pain management needs and perspectives of patients from Chinese backgrounds: a systematic review of the Chinese and English literature. Palliat Support Care. 2018;16(6)1-15.

37. Bowser D, Marqusee H, El Koussa M, Atun R. Health system barriers and enablers to early access to breast cancer screening, detection, and diagnosis: a global analysis applied to the MENA region. Public Health. 2017;152:58-74.

38. Duberstein PR, Chen M, Chapman BP, Hoerger M, Saeed F, Guancial E, et al. Fatalism and educational disparities in beliefs about the curability of advanced cancer. Patient Educ Couns. 2018;101(1):113-8.

39. Xu X, Luckett T, Wang AY, Lovell M, Phillips JL. Cancer pain management needs and perspectives of patients from Chinese backgrounds: a systematic review of the Chinese and English literature. Palliat Support Care. 2018;16(6):785-99.

40. Masel EK, Schur S, Watzke HH. Life is uncertain. death is certain. Buddhism and palliative care. J Pain Symptom Manage. 2012;44(2):307-12.

41. Wigginton B, Farmer K, Kapambwe S, Fitzgerald L, Reeves MM, Lawler SP. Death, contagion and shame: the potential of cancer survivors' advocacy in Zambia. Health Care Women Int. 2018;39(5):507-21.

42. Haozous EA, Knobf MT, Brant JM. Understanding the cancer pain experience in American Indians of the Northern Plains. Psychooncology. 2011;20(4):404-10.

43. Ehsani M, Taleghani F, Hematti S, Abazari P. Perceptions of patients, families, physicians and nurses regarding challenges in cancer disclosure: a descriptive qualitative study. Eur J Oncol Nurs. 2016;25:55-61.

44. Abazari P, Taleghani F, Hematti S, Ehsani M. Exploring perceptions and preferences of patients, families, physicians, and nurses regarding cancer disclosure: a descriptive qualitative study. Support Care Cancer. 2016;24(11):4651-9.

45. Shahid S, Taylor EV, Cheetham S, Woods JA, Aoun SM, Thompson SC. Key features of palliative care service delivery to Indigenous peoples in Australia, New Zealand, Canada and the United States: a comprehensive review. BMC Palliat Care. 2018;17(1):72.

46. Givler A, Maani-Fogelman PA. Cultural competence in pain and palliative care. Treasure Island: StatPearls; 2018.

47. Torresan MM, Garrino L, Borraccino A, Macchi G, De Luca A, Dimonte V. Adherence to treatment in patient with severe cancer pain: a qualitative enquiry through illness narratives. Eur J Oncol Nurs. 2015;19(4):397-404.

48. Efstathiou N, Vanderspank-Wright B, Vandyk A, et al. Terminal withdrawal of mechanical ventilation in adult intensive care units: a systematic review and narrative synthesis of perceptions, experiences and practices. Palliat Med. 2020;34:1140. 
49. Mendieta M, Buckingham RW. A review of palliative and hospice care in the context of Islam: dying with faith and family. J Palliat Med. 2017;20(11):1284-90.

50. Whitman SM. Pain and suffering as viewed by the Hindu religion. J Pain. 2007;8(8):607-13.

51. Chakraborty R, El-Jawahri AR, Litzow MR, Syrjala KL, Parnes AD, Hashmi SK. A systematic review of religious beliefs about major end-of-life issues in the five major world religions. Palliat Support Care. 2017;15(5):609-22.

52. Weiss RB. Pain management at the end of life and the principle of double effect: a Jewish perspective. Cancer Invest. 2007;25(4): 274-7.

53. Weir H. Expected new cancer cases and deaths in 2020. CDC. 2020. https://www.cdc.gov/cancer/dcpc/research/update-oncancer-deaths/index.htm. Accessed 8 Sept 2020.

54. UICC. Governments need to fulfill their commitments to universal health coverage. UICC. 2019. https://www.uicc.org/news/ governments-need-fulfil-their-commitments-universal-healthcoverage. Accessed 8 Sept 2020.

55. Ward ZJ, Scott AM, Hricak H, Abdel-Wahab M, Paez D, Lette MM, et al. Estimating the impact of treatment and imaging modalities on 5-year net survival of 11 cancers in 200 countries: a simulation-based analysis. Lancet Oncol. 2020;21(8):1077-88.

56. Horton R. A milestone for palliative care and pain relief. Lancet. 2018;391(10128):1338-9.

57. Clark J, Gardiner C, Barnes A. International palliative care research in the context of global development: a systematic mapping review. BMJ Support Palliat Care. 2018;8(1):7-18.

58. Clelland D, van Steijn D, Macdonald M, Connor S, Centeno C, Clark D. Global development of children's palliative care: the picture in 2017 [version 2; peer review: 2 approved with reservations, 1 not approved]. Wellcome Open Res. 2020;5:99.

$59 . \bullet$ Abu-Odah H, Molassiotis A, Liu J. Challenges on the provision of palliative care for patients with cancer in low- and middle-income countries: a systematic review of reviews. BMC Palliat Care. 2020;19(1):55 This systematic review focuses on low-tomiddle-income countries (LMICs), who have a disproportionate burden in lieu of the sharp growth of non-communicable diseases and lack of palliative care services. Challenges of palliative care integration include knowledge deficits of healthcare professionals and communities, inadequate workforces, and insufficient infrastructure. Understanding the challenges that exist will help develop a pathway for palliative care integration in LMICs.

60. Sedhom R, Gupta A, Von Roenn J, Smith TJ. The case for focused palliative care education in oncology training. J Clin Oncol. 2020;38(21):2366-8.

61. Spiker M, Paulsen K, Mehta AK. Primary palliative care education in U.S. residencies and fellowships: a systematic review of program leadership perspectives. J Palliat Med. 2020;23:1392.

62. Henderson JD, Boyle A, Herx L, Alexiadis A, Barwich D, Connidis $\mathrm{S}$, et al. Staffing a specialist palliative care service, a team-based approach: expert consensus white paper. J Palliat Med. 2019;22(11):1318-23.

63. Silbermann ME. Palliative care for chronic cancer patients: global approaches and future applications. Springer-Nature: Switzerland; 2020.

64. Nunes E, Santos HDS, Dutra GA, Cunha J, Szylit R. Soul care in the hospital nursing context: an analysis based on transpersonal caring. Rev Esc Enferm USP. 2020;54:e03592.

65. Kelly MM, Griffith PB. Umbrella review of school age health outcomes of preterm birth survivors. J Pediatr Health Care. 2020;34(5): e59-76.

66. Ameli R, Sinaii N, West CP, Luna MJ, Panahi S, Zoosman M, et al. Effect of a brief mindfulness-based program on stress in health care professionals at a US biomedical research hospital: a randomized clinical trial. JAMA Netw Open. 2020;3(8):e2013424.

67. Ben-Arye E, Portalupi E, Keshet Y, et al. Enhancing palliative care with mindful touch: impact of a manual and movement therapy training program in an international multidisciplinary integrative oncology setting. J Pain Symptom Manage. 2021;61(2):229-236.

68. Silbermann ME. Palliative care: the role and importance of research in promoting palliative care practices: reports from developed countries. Vol. 2. New York: NOVA Science Publishers; 2019.

69. Silbermann ME. Palliative care: the role and importance of research in promoting palliative care practices: reports from developing countries. Vol. 3. New York: NOVA Science Publishers; 2019.

Publisher's Note Springer Nature remains neutral with regard to jurisdictional claims in published maps and institutional affiliations. 